\title{
Numerical investigation on the flowfield of "swallowtail" cavity for supersonic mixing enhancement
}

\author{
C. Wang $\cdot$ Z. Jiang $\cdot$ Z. Hu $\cdot$ J. Lu
}

Received: 4 April 2008 / Revised: 22 September 2008 / Accepted: 13 October 2008 / Published online: 19 December 2008

(C) The Chinese Society of Theoretical and Applied Mechanics and Springer-Verlag GmbH 2008

\begin{abstract}
A "swallowtail" cavity for the supersonic combustor was proposed to serve as an efficient flame holder for scramjets by enhancing the mass exchange between the cavity and the main flow. A numerical study on the "swallowtail" cavity was conducted by solving the three-dimensional Reynolds-averaged Navier-Stokes equations implemented with a $k-\varepsilon$ turbulence model in a multi-block mesh. Turbulence model and numerical algorithms were validated first, and then test cases were calculated to investigate into the mechanism of cavity flows. Numerical results demonstrated that the certain mass in the supersonic main flow was sucked into the cavity and moved spirally toward the combustor walls. After that, the flow went out of the cavity at its lateral end, and finally was efficiently mixed with the main flow. The comparison between the "swallowtail" cavity and the conventional one showed that the mass exchanged between the cavity and the main flow was enhanced by the lateral flow that was induced due to the pressure gradient inside the cavity and was driven by the three-dimensional vortex ring generated from the "swallowtail" cavity structure.
\end{abstract}

Keywords Supersonic mixing - Flame holder . "Swallowtail" cavity · Combustion ignition · Numerical simulation

The project was supported by the National Natural Science Foundation of China (10602059, 10632090).

C. Wang $(\varangle) \cdot$ Z. Jiang $\cdot$ Z. Hu $\cdot$ J. Lu

Institute of Mechanics, Chinese Academy of Sciences,

100190 Beijing, China

e-mail: wangchun@imech.ac.cn

Z. Hu

ReCAPT, Gyeongsang National University, Jinju,

Kyeongnam 660-701, South Korea

\section{Introduction}

It is well known that supersonic mixing, combustion ignition and flame-holding have been the challenging topics in hypersonic researches for decades, especially for liquid hydrocarbon fuels. The methods for ignition and flame-holding in subsonic combustors are not generally applied to supersonic combustors because of their lower aerodynamic efficiency. Several concepts have been proposed to promote air/fuel mixing, combustion ignition and flame-holding in supersonic flows, such as swirling jets, the thin plate for fuel injection, cavities embedded in combustor walls, ramp injectors and stream-wise vortex generators [1-7]. Each concept has its own merits and limitations when used in the propulsion system of supersonic air-breathing vehicles; however better understanding on these topics was gained from these kinds of studies and the hypersonic research benefits.

For flame-holding in supersonic flows, the cavity embedded in combustor walls is regarded as a simple and effective structure for supersonic combustors. The pioneer work on supersonic flows over a cavity was reported by Krishnamurty in his doctoral thesis [8]. Since then many researchers have been attracted to this topic for decades. In the 1990s, the cavity was used as a flame holder in the supersonic combustor and related tests were carried out in the CIAM (Central Institution of Aviation Motors). Its performance for flameholding was well demonstrated. It is understandable that the low speed circumfluence region in the cavity plays an important role in flame-holding, and the combustion ignition in supersonic flows can be enhanced by the mass exchange that mainly depends on the self-sustained acoustics resonance between the flame holder and the main supersonic flow. This sort of acoustics resonance is influenced by shear layers, vortex structures, shock waves and turbulence. However, introducing a cavity into the supersonic combustor also results in 
gas dynamic drag increase and the total pressure loss, therefore, the study on the high performance cavity is of significant importance in order to get higher thrust provided by scramjets for hypersonic vehicles which is reasonable in theory.

Two-dimensional cavities were widely used in supersonic combustors for scramjet research, in which the flow in the cavity moved with a low lateral velocity, and the exchange of mass, momentum and energy between the cavity and the main supersonic flow was limited because the two-dimensional vortex structure in the cavity was self-closed. The self-closed vortex is useful for flame-holding, but the low mass exchange is not helpful for combustion ignition. Therefore, creating lateral flows in cavities to enhance the mass exchange rate is essential to improve the ignition efficiency in the condition that the gas dynamic drag and the total pressure loss can still be reasonable low. A promising research work is to apply a three-dimensional cavity into the supersonic combustor for scramjets. Torda and Patel [9] studied flow features of a triangular-shaped cavity; Davis [10] numerically investigated the cavity with a slight configuration change in its lateral direction and his results showed that there was little performance improvement on supersonic mixing because the lateral flow thus created is weak; Huang and Xu et al. [11] reported a performance study on a two-dimensional cavity with diagonal slots and the better performance gain was demonstrated.

In this paper, a new three-dimensional cavity, referred to as "swallowtail" cavity, was proposed to enhance the mass exchange in supersonic combustors by creating stronger lateral flows. Numerical investigation was carried out by solving the Reynolds-averaged Navier-Stokes equations and the $k-\varepsilon$ two-equation turbulence model based on the accepted governing equations to simulate turbulent mixing. Doing so is because that numerical simulations are now more and more important in studying supersonic mixing [12,13], as well as that the numerical work can give details which are difficult to be measured in experiments and moreover very helpful for the previous design of experiments. Physical models and numerical algorithms were validated first. Then several test cases were calculated to investigate into the mechanism underlying the cavity flows. Finally the cavity performance as a flame holder was discussed in detail.

\section{The "swallowtail" cavity and problem specification}

The three-dimensional cavity is schematically shown in Fig. 1 where the cross-section of the cavity in the symmetric plane is the same as the conventional cavities, but the cross-section area is gradually reduced to zero in $Z$-direction. The cavity configuration looks much like a swallow tail, therefore, is referred to as the "swallowtail" cavity. The top angle of the cavity is $30^{\circ}$ and the inclined angle of its back wall is also $30^{\circ}$, as labeled in Fig. 1. It is expected that a three-

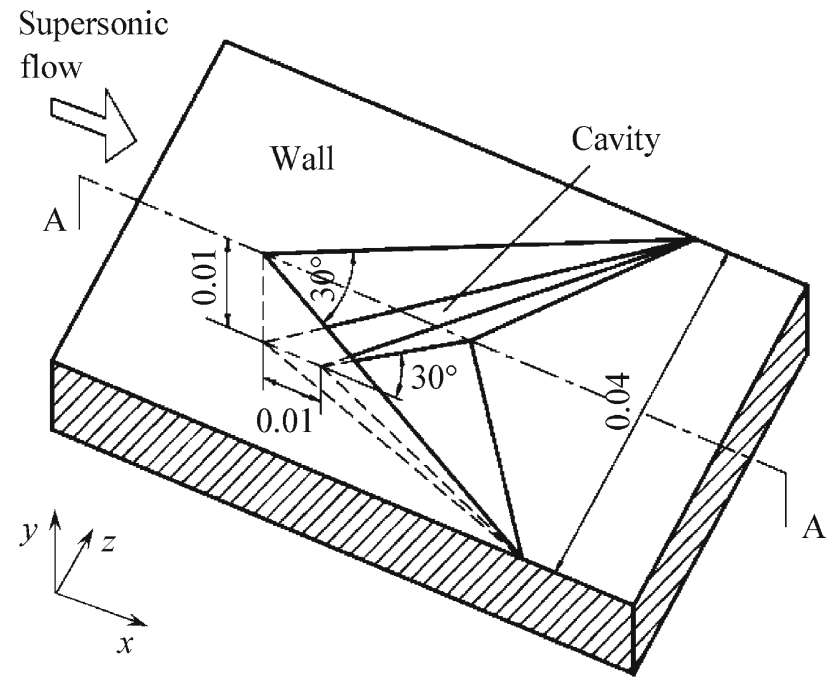

Fig. 1 Configuration of the "swallow-tail" cavity and problem specification: A-A: symmetric plane (unit: $\mathrm{m}$ )

dimensional vortex will be generated in the cavity when the supersonic flow sweeps over it. As a result of that, a strong lateral flow can be induced in the cavity for reasons that a low pressure region can be created in the top of the cavity, and the supersonic flow will be sucked into the cavity, moving toward combustor walls. Once the flow in the cavity goes out of the cavity at its lateral end, it will finally be mixed with the main supersonic flow. It is believed that the thus-created flow motion can enhance the mass exchange between the cavity and the main flow, and improve the cavity performance as a flame holder. The dimension of the "swallowtail" is illustrated in Fig. 1 in meter.

\section{Governing equations and numerical algorithm}

\subsection{Governing equations}

Considering the boundary layer instability, turbulent mixing, shock/boundary-layer interactions in the combustor cavity flow, the three-dimensional Reynolds-averaged NavierStokes equations for compressible fluid are applied for numerical investigation and written as

$\frac{\partial \boldsymbol{U}}{\partial t}+\frac{\partial \boldsymbol{F}}{\partial x}+\frac{\partial \boldsymbol{G}}{\partial y}+\frac{\partial \boldsymbol{H}}{\partial z}=\frac{\partial \boldsymbol{F}_{v}}{\partial x}+\frac{\partial \boldsymbol{G}_{v}}{\partial y}+\frac{\partial \boldsymbol{H}_{v}}{\partial z}+\boldsymbol{S}$,

where $\boldsymbol{U}$ is the conservative unknown variables, $\boldsymbol{F}, \boldsymbol{G}, \boldsymbol{H}$ are the convex fluxes, and $\boldsymbol{F}_{v}, \boldsymbol{G}_{v}, \boldsymbol{H}_{v}$ are the viscosity fluxes in $x, y, z$-directions, respectively, $\boldsymbol{S}$ stands for turbulence 
source term. These vector fluxes are defined as

$$
\begin{aligned}
\boldsymbol{U}= & {[\rho, \rho u, \rho v, \rho w, \rho E, \rho k, \rho \varepsilon]^{\mathrm{T}}, } \\
\boldsymbol{F}= & {\left[\rho u, \rho u^{2}+p, \rho u v, \rho u w,(\rho E+p) u, \rho k u, \rho \varepsilon u\right]^{\mathrm{T}}, } \\
\boldsymbol{G}= & {\left[\rho v, \rho u v, \rho v^{2}+p, \rho v w,(\rho E+p) v, \rho k v, \rho \varepsilon v\right]^{\mathrm{T}}, } \\
\boldsymbol{H}= & {\left[\rho w, \rho u w, \rho v w, \rho w^{2}+p,(\rho E+p) w, \rho k w, \rho \varepsilon w\right]^{\mathrm{T}}, } \\
\boldsymbol{F}_{v}= & {\left[0, \tau_{x x}, \tau_{x y}, \tau_{x z}, u \tau_{x x}+v \tau_{x y}+w \tau_{x z}\right.} \\
& \left.+q_{x},\left(\mu+\mu_{T} / \sigma_{k}\right) k_{x},\left(\mu+\mu_{T} / \sigma_{\varepsilon}\right) \varepsilon_{x}\right]^{\mathrm{T}}, \\
\boldsymbol{G}_{v}= & {\left[0, \tau_{y x}, \tau_{y y}, \tau_{y z}, u \tau_{y x}+v \tau_{y y}+w \tau_{y z}\right.} \\
& \left.+q_{y},\left(\mu+\mu_{T} / \sigma_{k}\right) k_{y},\left(\mu+\mu_{T} / \sigma_{\varepsilon}\right) \varepsilon_{y}\right]^{\mathrm{T}}, \\
\boldsymbol{H}_{v}= & {\left[0, \tau_{z x}, \tau_{z y}, \tau_{z z}, u \tau_{z x}+v \tau_{z y}+w \tau_{z z}\right.} \\
& \left.+q_{z},\left(\mu+\mu_{T} / \sigma_{k}\right) k_{z},\left(\mu+\mu_{T} / \sigma_{\varepsilon}\right) \varepsilon_{z}\right]^{\mathrm{T}}, \\
\boldsymbol{S}= & {\left[0,0,0,0,0, S_{k}, S_{\varepsilon}\right]^{\mathrm{T}}, }
\end{aligned}
$$

where $\rho, p, E$ denote density, flow pressure and total energy, and $u, v, w$ are the velocity components in $x, y, z$-directions, respectively. $K$ and $\varepsilon$ are the turbulence kinetic energy and the turbulence kinetic energy dissipation rate. $\tau_{x x}, \tau_{x y}, \tau_{x z}$, $\tau_{y z}$ are the shear stresses, and $q_{x}, q_{y}, q_{z}$ are the heat fluxes in $x, y, z$-directions, respectively. $S_{k}$ and $S_{\varepsilon}$ are the source terms of the turbulence kinetic energy and the turbulence kinetic energy dissipation rate. $\mu$ and $\mu_{T}$ are the laminar and turbulent viscous coefficients. $\sigma_{k}$ and $\sigma_{\varepsilon}$ are the constants of the turbulence model. The shear stresses and the heat fluxes are given by

$$
\begin{aligned}
\tau_{x x} & =\frac{2}{3}\left(\mu+\mu_{T}\right)\left(2 \frac{\partial u}{\partial x}-\frac{\partial v}{\partial y}-\frac{\partial w}{\partial z}\right)-\frac{2}{3} \rho k, \\
\tau_{y y} & =\frac{2}{3}\left(\mu+\mu_{T}\right)\left(2 \frac{\partial v}{\partial y}-\frac{\partial w}{\partial z}-\frac{\partial u}{\partial x}\right)-\frac{2}{3} \rho k, \\
\tau_{z z} & =\frac{2}{3}\left(\mu+\mu_{T}\right)\left(2 \frac{\partial w}{\partial z}-\frac{\partial u}{\partial x}-\frac{\partial v}{\partial y}\right)-\frac{2}{3} \rho k, \\
\tau_{x y} & =\tau_{y x}=\left(\mu+\mu_{T}\right)\left(\frac{\partial u}{\partial x}+\frac{\partial v}{\partial y}\right), \\
\tau_{y z} & =\tau_{z y}=\left(\mu+\mu_{T}\right)\left(\frac{\partial v}{\partial y}+\frac{\partial w}{\partial z}\right), \\
\tau_{x z} & =\tau_{z x}=\left(\mu+\mu_{T}\right)\left(\frac{\partial w}{\partial z}+\frac{\partial u}{\partial x}\right), \\
q_{x} & =\left(\lambda+\lambda_{t}\right) \frac{\partial T}{\partial x}, \\
q_{y} & =\left(\lambda+\lambda_{t}\right) \frac{\partial T}{\partial y} \\
q_{z} & =\left(\lambda+\lambda_{t}\right) \frac{\partial T}{\partial z} .
\end{aligned}
$$

In above expressions, $\lambda$ and $\lambda_{T}$ are the heat conductivity coefficients of laminar and turbulent flow, and $T$ is the fluid temperature. The state equation of ideal gas can be written as

$p=\rho R T$, where $R$ is the gas constant and taken to be $287.096 \mathrm{~J} /(\mathrm{kg} \mathrm{K})$ for air.

\subsection{The $k-\varepsilon$ two-equation turbulence model with the low-Reynolds modification}

The $k-\varepsilon$ two-equation turbulence model based on the Reynolds-averaged compressible Navier-Stokes equation is used in this paper to simulate the turbulent mixing flow. With this model the lower grid resolution is required than the large eddy simulation of turbulence. In the two-equation turbulence model, the source terms of $S_{k}$ and $S_{\varepsilon}$ in Eq. (1) are defined as the follows:

$$
\begin{aligned}
& S_{k}=P_{k}-\rho \varepsilon+E_{k}, \\
& S_{\varepsilon}=\left(f_{1} C_{\varepsilon 1} P_{k}-f_{2} C_{\varepsilon 2} \rho \tilde{\varepsilon}\right) T_{l}^{-1}+E_{\varepsilon},
\end{aligned}
$$

where $P_{k}$ is given as

$P_{k}=\left[\mu_{t}\left(\frac{\partial U_{i}}{\partial x_{j}}+\frac{\partial U_{j}}{\partial x_{i}}-\frac{2}{3} \frac{\partial U_{l}}{\partial x_{l}} \delta_{i j}\right)-\frac{2}{3} \rho k\right] \frac{\partial U_{i}}{\partial x_{j}}$.

The vortex viscosity coefficient is calculated with

$\mu_{t}=f_{\mu} C_{\mu} \rho k^{2} / \varepsilon$.

In Eqs. (3)-(5), $E_{k}, E_{\varepsilon}, f_{1}, f_{2}, C_{\varepsilon 1}, C_{\varepsilon 2}, f_{\mu}, C_{\mu}$ are the constants in the turbulence model. The Launder-Sharma (LS) turbulence model is used [14] in this paper, and the closure coefficients of the model $C_{\varepsilon 1}, C_{\varepsilon 2}, C_{\mu}, \sigma_{k}, \sigma_{\varepsilon}$ and $f_{1}, f_{2}$, $T_{l}, E_{k}, E_{\varepsilon}, f_{\mu}$ are specified in Tables 1 and 2 . The turbulent Reynolds number is defined as $R e_{t}=k^{2} /(v \varepsilon)$, where $v$ is the dynamic viscosity. $T_{l}$ is the coefficient for compressibility modification.

To overcome stiffness arising from the boundary conditions of the $k-\varepsilon$ turbulence model, the "point-implicit" method is applied, that is, the source terms of the turbulence model are implicitly solved with the following equation:

$(\boldsymbol{I}-\Delta t \cdot \boldsymbol{H})\left(\hat{\overline{\boldsymbol{U}}}^{n+1}-\hat{\overline{\boldsymbol{U}}}^{n}\right)=\Delta t \boldsymbol{S}^{n}$,

where $\boldsymbol{H}=\partial \boldsymbol{S} / \partial \boldsymbol{U}$, and can be expressed as

$$
\begin{aligned}
\boldsymbol{H}= & \boldsymbol{\Lambda}\left[\frac{2 \mu_{t}}{k}\left(\frac{\partial \boldsymbol{U}_{i}}{\partial x_{j}}+\frac{\partial \boldsymbol{U}_{j}}{\partial x_{i}}-\frac{2}{3} \frac{\partial \boldsymbol{U}_{l}}{\partial x_{l}} \boldsymbol{\delta}_{i j}\right) \frac{\partial \boldsymbol{U}_{i}}{\partial x_{j}}\right. \\
& \left.-\frac{2}{3} \boldsymbol{\delta}_{i j} \frac{\partial \boldsymbol{U}_{i}}{\partial U_{j}}, \frac{2}{3} \rho \boldsymbol{\delta}_{i j} \frac{\partial \boldsymbol{U}_{i}}{\partial \boldsymbol{U}_{j}} \cdot f_{1} C_{\varepsilon 1}-2 f_{2} \frac{\varepsilon}{k}\right] .
\end{aligned}
$$

Table 1 Closure coefficients for LS turbulence model

\begin{tabular}{lllll}
\hline$C_{\varepsilon 1}$ & $C_{\varepsilon 2}$ & $C_{\mu}$ & $\sigma_{k}$ & $\sigma_{\varepsilon}$ \\
\hline 1.44 & 1.92 & 0.09 & 1.0 & 1.3 \\
\hline
\end{tabular}


Table 2 Damping functions for LS turbulence model

\begin{tabular}{lllllll}
\hline$f_{1}$ & $f_{2}$ & $T_{l}$ & $f_{\mu}$ & $D$ & $E_{k}$ & $E_{\varepsilon}$ \\
\hline 1 & $1-0.3 \exp \left(-R e_{t}^{2}\right)$ & $k / \tilde{\varepsilon}$ & $\exp \frac{-3.4}{\left(1+R e_{t} / 50\right)^{2}}$ & $2 v\left(\frac{\partial \sqrt{k}}{\partial n}\right)^{2}$ & $2 v\left(\frac{\partial \sqrt{k}}{\partial x_{k}}\right)^{2}$ & $2 v v_{t}\left(\frac{\partial^{2} U}{\partial x_{k} \partial x_{l}}\right)^{2}$ \\
\hline
\end{tabular}

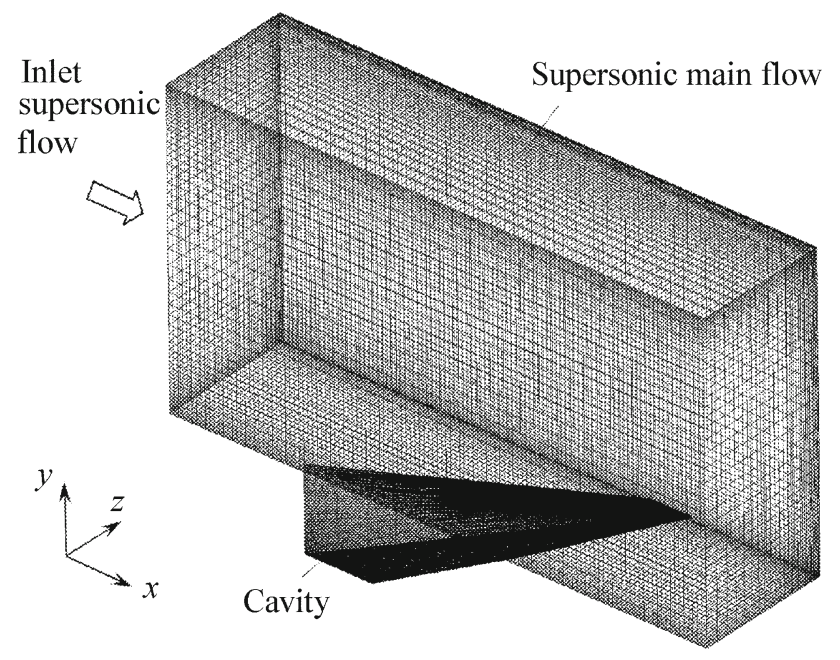

Fig. 2 The multi-block mesh system distributed over the half of the computational domain

\subsection{Numerical algorithm of finite difference method}

The governing equations are solved with the 2 nd order NND scheme of the finite difference method [15], and viscous terms are calculated with the 2 nd order central differencing. The convective terms of the governing equations in each coordinate direction can be differentiated as

$$
\boldsymbol{F}_{\text {convetive }}=\boldsymbol{F}_{i+\frac{1}{2}, L}^{+}-\boldsymbol{F}_{i-\frac{1}{2}, L}^{+}+\boldsymbol{F}_{i+\frac{1}{2}, R}^{-}-\boldsymbol{F}_{i-\frac{1}{2}, R}^{-},
$$

where

$\boldsymbol{F}_{i+\frac{1}{2}, L}^{+}=\boldsymbol{F}_{i}^{+}+\frac{1}{2} \min \bmod \left\{\Delta \boldsymbol{F}_{i-\frac{1}{2}}^{+}, \Delta \boldsymbol{F}_{i+\frac{1}{2}}^{+}\right\}$,

$\boldsymbol{F}_{i+\frac{1}{2}, R}^{-}=\boldsymbol{F}_{i+1}^{-}+\frac{1}{2} \min \bmod \left\{\Delta \boldsymbol{F}_{i+\frac{1}{2}}^{-}, \Delta \boldsymbol{F}_{i+\frac{3}{2}}^{-}\right\}$,

where $\Delta \boldsymbol{F}^{+}$is the forward difference and $\Delta \boldsymbol{F}^{-}$denotes the backward difference. The minmod function is defined as

$\min \bmod (x, y)=\operatorname{sgn}(x) \cdot \max \{0, \min [|x|, y \operatorname{sgn}(x)]\}$.

The computational domain is taken to be half of the physical region of interest, as shown in Fig. 2. A multi-block, overlapped mesh system is used in the present numerical simulation. There are two mesh blocks, as shown in Fig. 2. The grid size in $z$-direction of the two blocks is equal to each other. In the overlapped grid, the data calculated in each time step are exchanged between the two blocks with area-weighted interpolation for the next iteration. The grid node number in the main flow region is $121 \times 35 \times 31$, and that in the cavity is $61 \times 35 \times 31$. The grid nodes are distributed more near the wall to get higher resolution solutions for boundary layer and the minimal grid size near walls is about $1.2 \times 10^{-4} \mathrm{~m}$.

\subsection{Initial and boundary conditions}

Assuming that the flow over the cavity shown in Fig. 1 is symmetric, all variables on the symmetric plane are specified with the mirror-reflection boundary condition. The inlet boundary is taken to be the supersonic flow at Mach number 2 considering which as the inflow condition of scramjet combustors. The outflow boundary is given by extrapolation implemented with a low pressure to avoid the downstream perturbation influence. As for the top boundary, all variables are extrapolated from the internal flow field with a non-reflection requirement. On the bottom wall, the non-slip boundary is applied with the adiabatic condition for temperature. As the boundary conditions for the cavity flow, all the variables in its boundaries are exchanged from the main flow field. On the symmetric plane, the mirror-reflection condition is applied to all variables. Other boundaries are taken to be the non-slip wall and adiabatic conditions.

Initially for the present numerical simulation, the coming supersonic flow was specified with Mach number $M=2.0$, the static pressure $p_{0}=100 \mathrm{kPa}$, and the static temperature $T_{0}=300 \mathrm{~K}$. The other part of the flow field is given with the same static pressure and temperature. The explicit time marching method is used to approach the quasi-steady solutions.

\subsection{Physical model validation and code verification}

The first case is selected to validate the turbulence model. The physical model is a dual-combustor of scramjets as shown in Fig. 3. The detailed experimental result [16] can be obtained. In this test case, the air flow comes from the heater and enters into the supersonic combustor at Mach number $M_{2}=2.09$ with static pressure $p_{2}=0.0977 \mathrm{MPa}$ and temperature $T_{2}=$ $491.0 \mathrm{~K}$. The hot gas flow comes from the subsonic combustor and enters into the supersonic combustor through a nozzle at Mach number $M_{2}=1.25$ with static pressure $p_{1}=$ $0.1731 \mathrm{MPa}$ and temperature $T_{2}=1771.9 \mathrm{~K}$. Two supersonic flows are mixed together in the combustor, and then the ignition takes place. Pressure variations along combustor walls are recoded to monitor combustion processes during both the experiment and CFD simulations. 


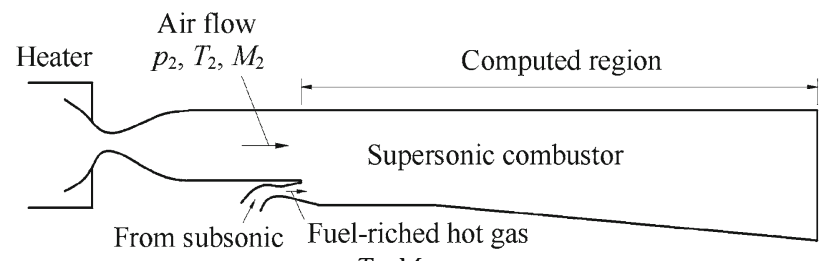

combustor $\quad p_{1}, T_{1}, M_{1}$

Fig. 3 The test case of the dual-combustor for scramjets

Numerical results are plotted in Fig. 4 with the experimental data together for comparison. Figure 4a shows the pressure along the upper wall and Fig. $4 \mathrm{~b}$ denotes the pressure along the lower wall. From these two figures it is observed that the computed pressure with the turbulence model agrees well with the experimental data. The $k-\varepsilon$ two-equation turbulence model works better than the B-L turbulence model embedded with Prentle mixing length does. Effectiveness of the applied turbulence model was well demonstrated.

The second case was carried out for verification of the numerical code. The model is a conventional cavity for supersonic combustors. The experiment was conducted by Yi et al. with a supersonic wind tunnel at University of National Defense Science and Technology, China. The Mach number is taken to be 3.05 with the total temperature $T_{t 0}=300 \mathrm{~K}$ and total pressure $p_{t 0}=101.6 \mathrm{kPa}$. The cavity flow was visualized with the NPLS (Nano-based Planar Laser Scattering) technology in which nano-particles are used as tracers to demonstrate density variations in flow fields. The denser the particle distributes, the higher the density variation there is. Numerical simulations for the two-dimensional cavity were carried out using the present three-dimensional numerical code with the same inflow conditions as that in the experiment. Both the numerical results and the experimental picture are presented in Fig. 5 for comparison.
The numerical density contours and streamlines in the symmetric plane of the cavity are plotted in Fig. 5a. The experimental picture showing the flow on the same plane is presented in Fig. 5b. From these results, it is observable that the shock location, shear layer development and the low density area in the cavity look similar to each other, and therefore, the numerical code was well verificated.

\section{Numerical results and discussion}

After solution validation and code verification, three-dimensional cavity flows were simulated to investigate physical mechanisms that affect the performance of the "swallowtailtype" cavity as a flame holder. Figure 6a shows non-dimensional pressure $\left(p / p_{0}\right)$ and Mach number distributions of the flow field in the symmetric plane. Figure $6 \mathrm{~b}$ shows these in the plane near the end of the cavity. From these figures, it can be seen that the flow field exhibits a strong three-dimensional feature because the pressure distributions are obviously different at different locations in the cavity. In the symmetric plane, there is a low pressure region in which the gas is sucked into the cavity from the main flow and a lower shear layer develops, as shown in Fig. 6a. The pressure at the end of the cavity is higher than that in the main flow, so that the gas in the cavity can be driven out into the main flow, which results in the shear layer rising higher, as shown in Fig. 6b. The shock wave induced by the cavity appears to be stronger in the symmetric position, but weaker at the end of the cavity. This phenomenon indicates that the vortex generated in the symmetric plane is much stronger than that in other locations, which demonstrates that the three-dimensional vortex does exist in the "swallowtail" cavity. Furthermore, it is the three-dimensional vortex that induces a transverse flow along the cavity which is helpful to promote supersonic mixing and
Fig. 4 Comparison of pressure distributions numerically predicted along combustor walls with experimental results [16]. a Pressure distribution on the upper wall; b Pressure distribution on the lower wall
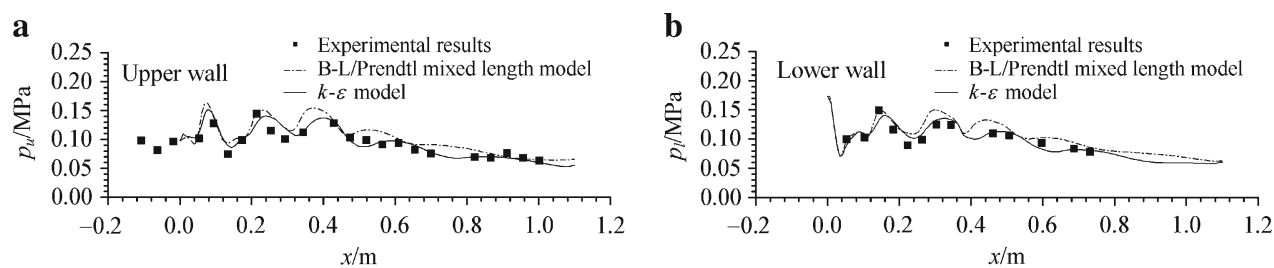

Fig. 5 Comparison between CFD and experiments of two-dimensional cavity flows with Mach number of 3.05 . a Density contours and streamlines of CFD results; b NPLS photo from flow visualization
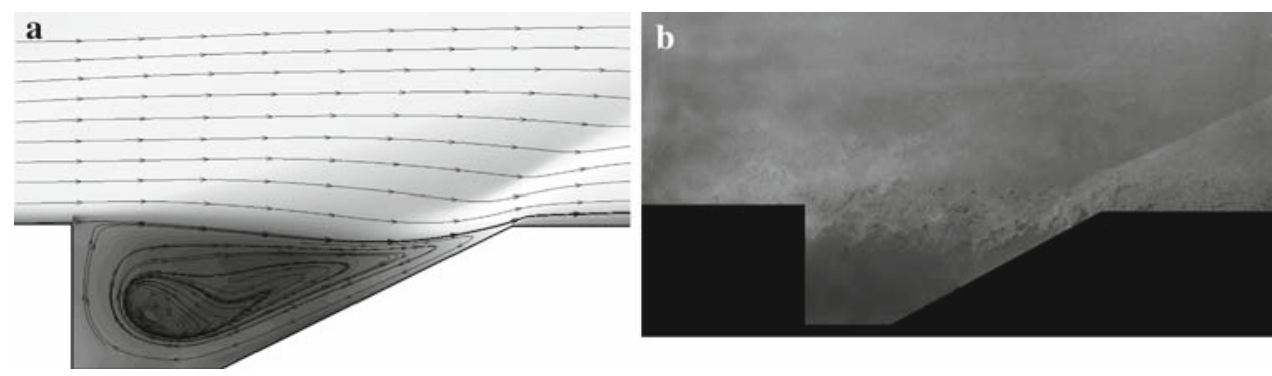
Fig. 6 The non-dimensional pressure $\left(p / p_{0}\right)$ and local Mach number distributions. a Pressure (left) and Mach number (right) distributions of the flow filed in the symmetric plane; $\mathbf{b}$ Pressure (left) and Mach number (right) distributions of the flow filed near the end of the cavity
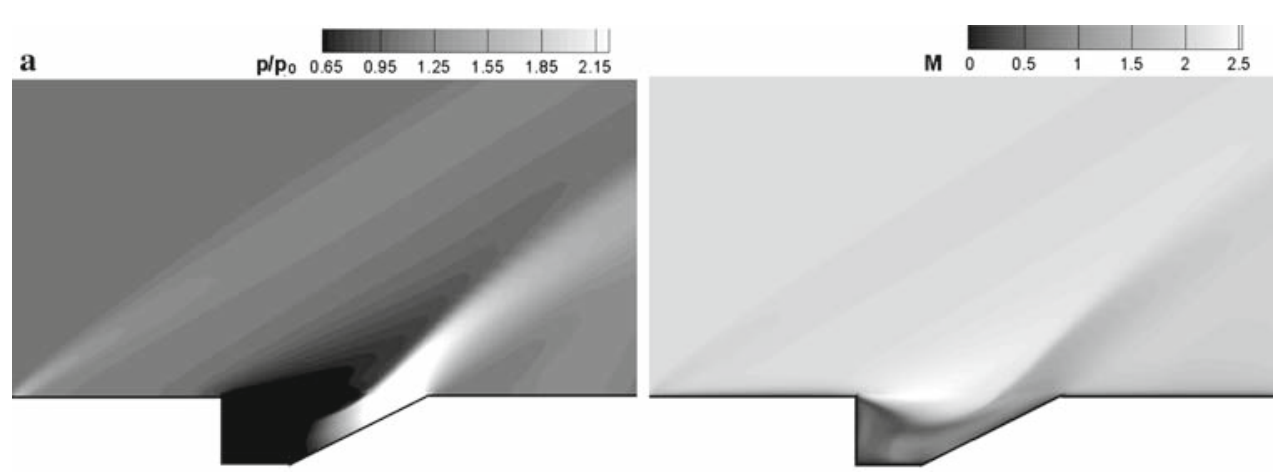

b

$\begin{array}{lllllll}\mathbf{p}^{\prime} \mathbf{p}_{0} & 0.65 & 0.95 & 1.25 & 1.55 & 1.85 & 2.15\end{array}$
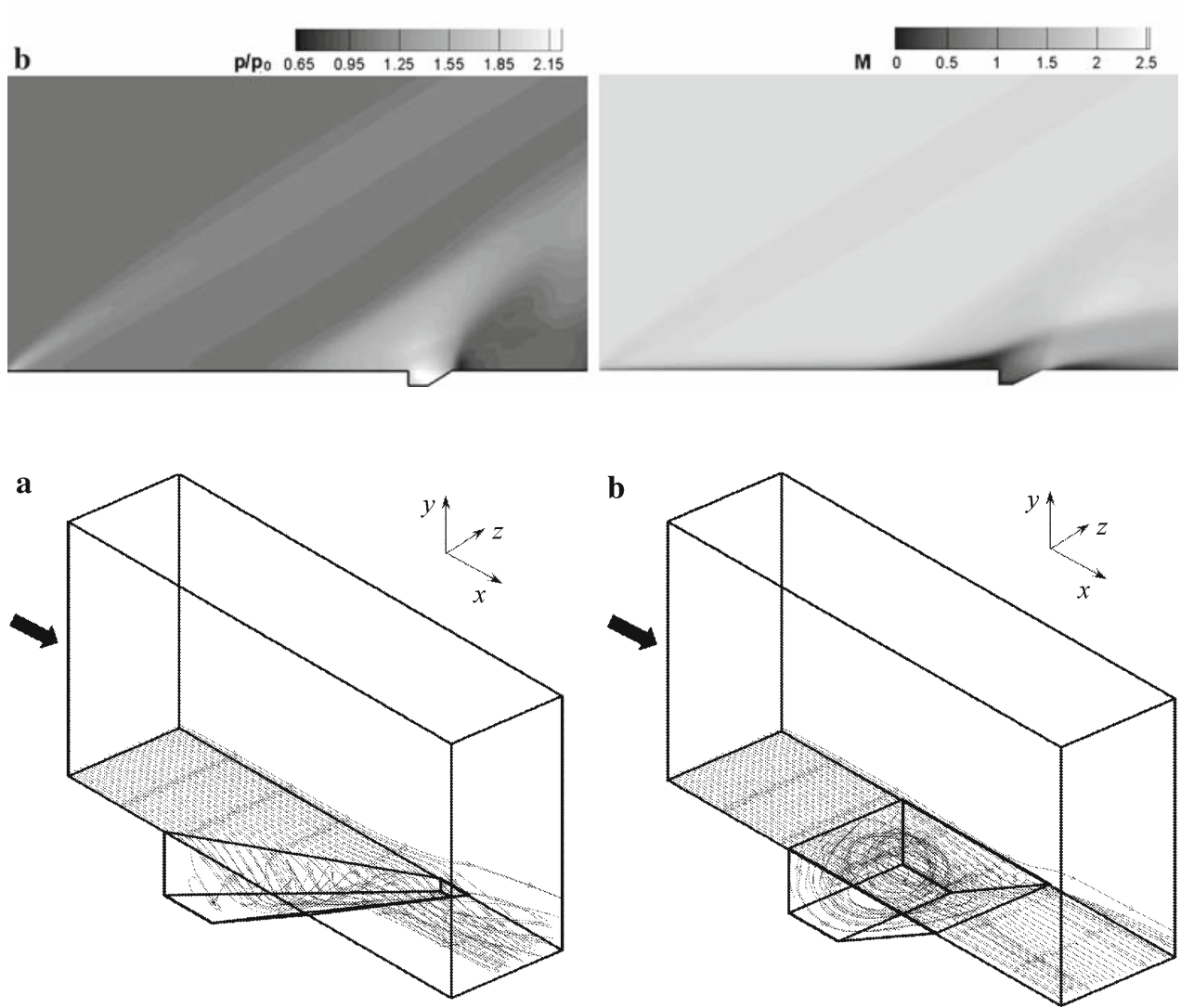

Fig. 7 Streamlines starting from the upstream boundary layer in two numerical test cases for comparison. a Streamlines in the "swallowtail" cavity flow; b Streamlines in the conventional cavity enhance the mass exchanges between the cavity and the main supersonic flow. In addition, shock-induced perturbations are also helpful for supersonic mixing.

It is well known that the low speed circumfluence region in a cavity embedded into a combustor wall can act as a flame holder and the mass exchange rate of the hot gas from the cavity into the main flow stands for the flame holder performance. The results above show that the "swallowtail" cavity actually enhances the mass exchange, therefore, it is a promising configuration as a flame holder for scramjet.

Streamlines starting from the upper stream boundary layer were plotted in Fig. 7a where the arrows stand for the local flow direction. It can be seen that the flow is trapped into the cavity, moves along the cavity toward combustor walls, and then spirally goes out of the cavity at its lateral end. This kind of flow feature with the "swallowtail" cavity is very helpful to promote the mass exchange between the cavity and the main supersonic flow. For comparison, the streamlines starting from the upper stream boundary layer in a case with a two-dimensional cavity is also presented in Fig. 7b. Inflow conditions and the geometric sizes in the symmetric plane are the same as those in the case with the "swallowtail" cavity. It is observed that the gas flow in this type of cavity is rather enveloped. Obviously, the "swallowtail" cavity is more efficient for enhancing the mass exchange between the cavity and the main flow than the conventional cavity.

Figure 8a shows non-dimensional pressure distributions on the bottom wall of the "swallowtail" cavity, where the non-dimensional pressure $p / p_{0}$ is defined as the ratio of the local pressure to the inflow pressure. In the symmetric plane, 
Fig. 8 The pumping effect and the particular flow pattern generated from the "swallowtail" cavity. a Pressure distributions on the combustor bottom wall; b Flow pattern generated due to the "swallowtail" cavity

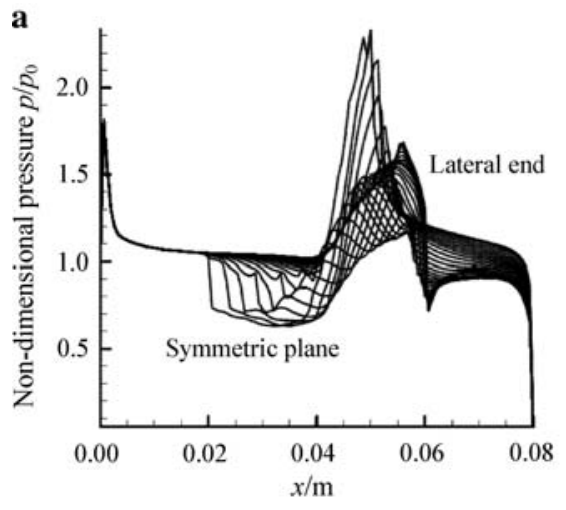

b

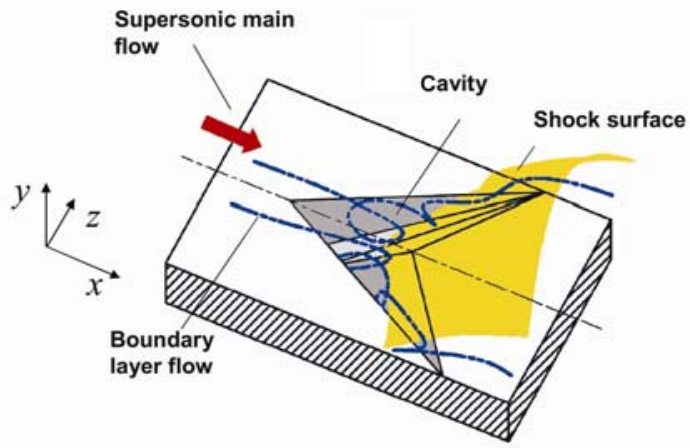

the pressure on the bottom wall of the cavity is lower than that in the main flow. So the pressure difference helps the cavity to suck the gas from the main flow. At the lateral end of the cavity, the pressure is higher than that in the main flow so that it is helpful to drive the gas in the cavity into the main flow. The process of this physical phenomenon is the so-called "pumping effect" of the "swallowtail" cavity, which derives from the three-dimensional vortex generated in the cavity. If the vortex can be organized effectively, the pumping phenomenon will be utilized to enhance the mass exchange between the cavity and the main supersonic flow so that the more efficient flame holder could be designed. The pressure rising downstream in the cavity is clear which is believed to be induced by shock waves. The high pressure at this location drives the transverse flow to move along the cavity and the three-dimensional vortex is thus generated.

By summarizing what was discussed before, the flow pattern particularly generated due to the "swallowtail" cavity is schematically shown in Fig. 8b. The gas in the main supersonic flow near the symmetric plane is trapped into the cavity, spirally moves along the cavity in the lateral direction, then rushes out at the end of the cavity, and finally be mixed efficiently with the main flow with the further help of shock waves, as shown in Fig. 8b. Such the flow motion develops into a three-dimensional vortex which promotes the mass exchange between the cavity and the main flow, therefore the flame holder performance will benefit.

\section{Concluding remarks}

The mechanism of the three-dimensional flow motion generated by the "swallowtail" cavity was numerically investigated by solving the three-dimensional Reynolds-averaged Navier-Stokes equations implemented with a turbulence model in a multi-block mesh system. It is observed in the numerical results that the "pumping effect" exists in the three-dimensional flow motion due to the "swallowtail" cavity configuration. Because of the "pumping effect", more gas from the main supersonic flow is trapped into the cavity where the gas moves transversely along the cavity, then flows out at its lateral end, and finally is efficiently mixed with the main flow. The flow mechanism in this phenomenon is very useful to enhance the supersonic mixing between the cavity and the main supersonic flow. The present study demonstrated that the "swallowtail" cavity works well as an effective flame holder for scramjets because it could provide with a low speed circumfluence region as well as the intensive mass exchange rate between the cavity and the main flow.

Acknowledgments The authors would like to thank Prof. Yi Shihe of University of National Defense Science and Technology for his generous permission to use one of his experimental photos.

\section{References}

1. Curran, D.T., Heiser, W.H., Pratt, D.T.: Fluid phenomena in scramjet combustion systems. Ann. Rm. Fluid. Mech. 28, 323-360 (1996)

2. Kraus, D.K., Cutler, A.D.: Mixing enhancement by use of swirling jets. AIAA Paper 93-3126 (1993)

3. Gouskov, O., Kopchenov, V., Vinogradov, V., Waltrup, P.J.: Numerical researches of gaseous fuel pre-injection in hypersonic 3-D inlet. AIAA Paper 2000-3599 (2000)

4. Ben-Yakar, A., Hanson, R.K.: Cavity flame-holders for ignition and flame stabilization in scramjets: an overview. J. Propulsion Power 17(4) (2001)

5. Donohue, J.M., McDaniel, J.C. Jr., Haj-Hariri, H.: Experimental and numerical study of swept ramp injection into a supersonic flowfield. AIAA Paper 93-2445 (1993)

6. Eklund, D.R., Gruber, M.R.: Study of a supersonic combustor employing and aerodynamic ramp pilot injector. AIAA Paper 992249 (1999)

7. Wei, M.J., Sun, D.J., Yin, X.Y., et al.: Stability analysis on supersonictrailing-line vortex. Acta Mech. Sin. 31(6), 694-699 (in Chinese) (1999)

8. Karamcheti, K.: Sound radiation from surface cutouts in high speed flow. Ph.D. Thesis, California Institute of Technology (1956)

9. Torda, T.P., Patel, B.R.: Analytical and experimental investigations of oscillations in rocket motor baffle cavities. NASA Tech Report, AD0849511

10. Davis, D.L.: Numerical analysis of two and three dimensional recessed flame holders for scramjet applications. Ph.D. Thesis, ADA324246 
11. Huang, S.H., Xu, S.L., Liu, X.Y.: Numerical investigation on two phase flow of a kerosene-fueled scramjet with 3D cavity (I) Numerical calibration and characteristics of general flow. J. Propulsion Technol. 25(6) (2004) (in Chinese)

12. Hu, Z.M., Lü, J.M., Jiang, Z.L., et al.: Numerical study on the performance of nozzle flow for supersonic chemical oxygen-iodine lasers. Acta Mech. Sin. 24(2), 133-142 (2008)

13. Chen, J.Q., Zhuang, F.G.: Numerical simulation of supersonic reacting mixing layer. Acta Mech. Sin. 13(2), 97-105 (1997)
14. Du, T., Wu, Z.N.: Mixed analytical/numerical method for lowReynolds-number $k-\varepsilon$ turbulence models. AIAA J. 42(6), 11401153 (2004)

15. Zhang, H.X.: Non-oscillatory and non-free-parameter dissipation difference scheme. Acta Aerodyn. Sin. 5(2), 143 (in Chinese) (1988)

16. Wang, C., Lu, H.P., Ming, S.T.: Numerical predictions of cold flowfield of supersonic combustor in dual-combustor ramjet. J. Propulsion Technol. 20(5), 35-38 (in Chinese) (1999) 